\title{
Schlangen, Studenten und ein Bier - ein Problemaufriss
}

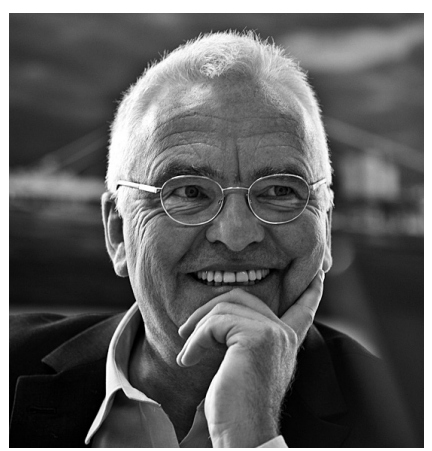

VON BERND HALFAR

Prof. Dr. Bernd Halfar lehrt

Management in sozialen

Einrichtungen und Organisationsentwicklung an der Katholischen Universität Eichstätt-Ingolstadt. Er ist Mitgründer und Partner der Unternehmensberatung xit. forschen.planen.beraten $\mathrm{GmbH}$ mit Büros in Nürnberg und Berlin. Er ist Mitglied des Beirats der Zeitschrift SOZIALwirtschaft und des Informationsdienstes SOZIALwirtschaft aktuell. E-Mail

bernd.halfar@ku-eichstaett.de

\author{
Das System sozialer Dienstleistungen in Deutschland \\ ist auf einem Expansions- und Qualitätstrip - \\ und benötigt hierfür entsprechend immer mehr \\ finanzielle Ressourcen. Das ist einer der Gründe, \\ weshalb die Finanzierung des Sozialwesens \\ überprüft werden muss - doch nach welchen \\ Kriterien? Elf Aspekte gilt es zu berücksichtigen, \\ will man nicht vom Regen in die Traufe kommen.
}

Zum Semesterbeginn hatte ich in einem Seminar für Zweitsemester der Sozialarbeit nach dem Branchenumsatz der deutschen Sozialwirtschaft gefragt. Wessen Schätzung der Wahrheit am nächsten lag, sollte eine Halbe Bier bekommen. Der Zuschlag fiel auf die höchste Schätzung von vier Millionen Euro.

Das Gute daran ist, dass ich jetzt in der späteren Vorlesung ein gutes Beispiel für das Theorem der fiskalischen Illusion habe. Demnach unterschätzen die steuerzahlenden Bürger systematisch die finanziellen Dimensionen staatlicher Programme. Das Schlechte daran ist, dass alle Abiturienten, die gute Einkommen haben wollen, nicht in eine Branche gehen, die lediglich vier Millionen Euro umsetzt.

Wenn es überhaupt keinen Sozialarbeiter in Deutschland geben würde, nur die 200 Erstsemester aus Eichstätt, bekäme jeder 20.000 Euro im Jahr. Allerdings gäbe es dann keinen einzigen Klienten, weil das Geld ja komplett für die Lohnsumme aufgebraucht würde.

Je nach Abgrenzungskriterien liegt das Branchenvolumen der Sozialwirtschaft in Deutschland zwischen 80 und 100 Milliarden Euro.

Seit vielen Jahren sind einige Entwicklungen $\mathrm{zu}$ beobachten: Es fließt immer mehr Geld in die Sozialarbeit, die Branche wächst und wächst, es werden immer mehr Mitarbeiter beschäftigt, immer mehr Berufsgruppen werden akademisiert, es gibt immer mehr Klienten, der Anteil der Steuerfinanzierung an den Gesamtausgaben wächst, weil auch beim Finanzierungsträger »Sozialversicherung « der Anteil der »Staatsfinanzierung « demografisch bedingt die Bedeutung der umlagefinanzierten Beiträge relativiert und die vereinbarten die Qualitätsstandards steigen kontinuierlich. Das System sozialer Dienstleistungen ist auf einem Expansions- und Qualitätstrip - und benötigt hierfür entsprechend immer mehr finanzielle Ressourcen.

Unter der Bedingung des Kostenerstattungsprinzips würden sich jetzt die staatlichen Kassen entsprechend öffnen - und wir hätten kein Finanzierungsthema. Nun ist ja das Kostenerstattungsprinzip in den Jahren $1994 \mathrm{ff}$. nicht grundlos in leistungsorientierte Finanzierungsformen umgewandelt worden. Mit diesen leistungsorientierten Finanzierungsformen war die Hoffnung auf eine Verbesserung der allokativen Effizienz, der Rationalität und Steuerungsfähigkeit des Systems verknüpft. Durch Leistungs- und Kostenorientierung der Träger sollte die betriebliche Effizienz gesteigert werden, und in der Folge entstehende Überschüsse ebenso beim Träger verbleiben wie das Auslastungsrisiko. 
Das mit dem Risikoübertrag hat geklappt, das mit dem Gewinnen nicht so richtig. Die Branche, ganz deutlich zu beobachten in der Altenpflege, geht in die Rücklagen, betreibt auf der Immobilienseite Substanzverzehr und kann die sich öffnende Schere zwischen laufenden Personal- und Betriebskosten und Entgelten nicht schließen. Die öffentliche Hand weigert sich, einen kostentreibenden Begriff von Strukturqualität mit definierten Personalstandards und Personalmengen aufzugeben, und sie weigert sich, Tariferhöhungen als Argument in den Preisverhandlungen zu akzeptieren.

Es hat den Anschein, dass hinter den Rücken der Beteiligten während des Spiels die Regeln verändert wurden. Dass die Finanzierung sozialer Einrichtungen und Dienste ein Suchprozess ist, der immer wieder neu justiert werden muss, dass jede Finanzierungsform nicht nur Wirkungen, sondern auch Nebenwirkungen hat, dass auch die Entwicklung der Steuereinnahmen und der Klientenzahlen beim »Pricing « eine Rolle spielen müssen, ist auch klar.

Alle wissen alles, und insbesondere, dass die Finanzierung des Sozialwesens überprüft werden muss. Doch welche Kriterien sollten Finanzierungsformen erfüllen? Hierzu elf Kriterien zur Diskussion.

\section{Erstes Kriterium: Mengenexpansion}

Erinnert sei an die kleine Geschichte der indischen Giftschlangenplage. Die indische Regionalregierung will die Schlangenplage in den Griff be- kommen und zahlt für jede abgelieferte getötete Giftschlange eine Prämie von $\mathrm{x}$ Rupien. Es entstehen überall Schlangenzuchtfarmen ... Für dieses "Cobra-Theorem " lassen sich auch im deutschen Sozialwesen viele Belege finden. Hier stellt sich die alte Frage nach dem »richtigen Bedarf «, aber es stellt sich auch die Frage nach diagnostischen Moden in der Sozialarbeit und, wie beim DRG-System im Krankenhaus, nach den Skaleneffekten durch Mengengenerierung.

\section{Zweites Kriterium: Quality Skimping}

Im Dienstleistungsbereich, die ServiceBlueprintmodelle zeigen das ja sehr schön, geschehen im Dienstleistungsprozess manche Schritte »backstage«, für

\section{Wie sich die deutsche Sozialwirtschaft finanziert}

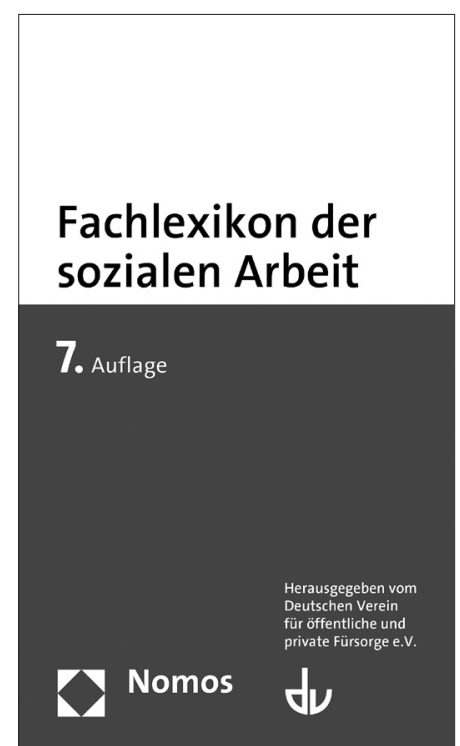

Die Finanzierung sozialer Dienste und Einrichtungen richtet sich nach der Trägerschaft, dem Selbstverständnis sowie den konkreten rechtlichen und wirtschaftlichen Rahmenbedingungen. Typische Finanzierungsformen sind in der Reihenfolge ihrer Bedeutung:

(1) Leistungsentgelte auf gesetzlicher oder vertraglicher Grundlage, die von der öffentlichen Hand oder den Nutzer/innen selbst, ggf. auch anteilig, gezahlt werden. Ermittelt wurden die Entgeltsätze in der Vergangenheit vorrangig inputorientiert nach dem Selbstkostendeckungsprinzip auf Basis der echten Selbstkosten. Inzwischen werden die Entgelte auf der Basis der in der Vergangenheit angefallenen Kosten ausgehandelt. Pro- blematisch ist hierbei ein undifferenzierter Rückgriff auf Betriebsvergleiche, da hierbei die Abhängigkeit zwischen verschiedenen Kostenpositionen ausgeblendet wird (z. B. Ersatz von Personalkosten durch Sachaufwand oder umgekehrt). In Teilbereichen werden die Entgeltverhandlungen inzwischen durch Ausschreibungen ersetzt. Dies führt zu einer Ausdünnung des bisherigen Angebots, wenn nur ein Teil der bisherigen Komplexleistungen in die Ausschreibung aufgenommen wird. Zum Beispiel kann eine undifferenzierte Ausschreibung und Neuvergabe des Rettungsdienstes zu gravierenden Qualitätseinbußen beim Katastrophenschutz des bisherigen Trägers führen.

(2) Zuwendungen der öffentlichen Hand wie Europäische Union, Bund, Länder, Landschaftsverbände und Kommunen. Die Vergabe erfolgt auf haushaltsrechtlicher Grundlage in der Regel durch Bewilligungsbescheid. Durch Bedingungen und Auflagen soll nach kameralistischem Verständnis der wirtschaftliche Mitteleinsatz sichergestellt werden; gar nicht so selten führt dies aber zu unnötigen Zusatzkosten. Wegen ihrer Input-Orientierung verliert diese Finanzierungsform an Bedeutung.

(3) Bei dem Dualen Finanzierungssystem werden die Investitionen durch Zuwendungen und die Betriebskosten durch Leistungsentgelte aufgebracht. Wegen der in Teilsegmenten möglichen Substitution von Investitionskosten durch Betriebskosten und umgekehrt, z. B. Wärmedämmung statt Heizkosten oder Maschinen statt Arbeitskraft, verlockt diese Finanzierungs- form zur unwirtschaftlichen Kostenverlagerung auf spätere Jahre oder andere Finanzierungsträger. Ein Beispiel hierfür ist die Krankenhausfinanzierung: Während die Krankenversicherungen die Betriebskosten tragen, übernehmen die Bundesländer die Investitionskosten.

(4) Mit Zeitspenden, also ehrenamtlichem Arbeitseinsatz, werden insbesondere anderweitig nicht oder nicht ausreichend finanzierbare Sozialleistungen erbracht.

(5) Spenden, Mitgliedsbeiträge und Erbschaften tragen in erster Linie bei gemeinnützigen Organisationen (Gemeinnützigkeit von Körperschaften) zur Finanzierung sozialer Aufgaben bei. Bei der Investitionsfinanzierung wird zunehmend auf neue Finanzierungsformen zurückgegriffen, wie Leasing oder Public Private Partnership. Hierbei sind die unter Outsourcing und Privatisierung beschriebenen Interessengegensätze zu beachten. In letzter Zeit werden auch gemeinnützigen Körperschaften mezzanine Finanzierungsformen angeboten. Die Finanzierung befindet sich in einem grundlegenden Wandel mit gravierenden Auswirkungen auf Zusammenstellung und Ausgestaltung der sozialen Angebote, die Anbieterstruktur und die gesamtgesellschaftliche Prosperität.

Thomas von Holt

Quelle: Deutscher Verein für öffentliche und private Fürsorge e. V. (Hg.): Fachlexikon der sozialen Arbeit. 7. Auflage. Nomos Verlagsgesellschaft, Baden-Baden 2011. 1.139 Seiten. 44,- Euro. ISBN 978-3-8329-5153-5. 
den Klienten unsichtbar, und somit auch vom Klienten nicht bewertbar. Durch manche Finanzierungsformen wird die Gefahr verstärkt, diese unsichtbare Leistungsqualität abzusenken.

\section{Drittes Kriterium: Cream Skimming}

Finanzierungsformen müssen auch danach bewertet werden, ob sie den Einrichtungen ermöglichen, ein $»$ KlientenPicking « zu betreiben. Man schöpft sozusagen den Rahm vom Topf und überlässt durch Cream Skimming den »schlechten Klienten« dem Wettbewerb. Alle wollen die "guten Klienten «, die entweder einen guten Deckungsbeitrag bringen oder, bei wirkungsorientierter Finanzierung, eine gute Sozialprognose haben.

\section{Viertes Kriterium: Rent Seeking}

Rent Seeking entsteht durch die Verteidigung ineffizienter Organisationsteile, die eigentlich nicht wettbewerbsfähig sind. Um diese weiterbetreiben zu können, müssen ineffiziente Organisationsteile durch Politikbeeinflussung dem Wettbewerbsgeschehen entzogen und/ oder subventioniert werden. Der hiefür notwendige Ressourcenanteil, die Rent Seeking Rate, wird der Dienstleistungsproduktion entzogen und mindert die Dienstleistungsproduktivität.

\section{Fünftes Kriterium: Paradoxe Effekte}

Manche Finanzierungsregelungen flüstern dem Dienstleister geradezu ins Ohr: "Erreiche das Gegenteil deiner eigentlichen Ziele." Angesprochen sind hier Rehabilitationserfolge, Erfolge in der Familienhilfe, die mit Abbruch der Fachleistungsstunden bestraft werden oder die Vermittlung leistungsfähiger Mitarbeiter in den ersten Arbeitsmarkt. Bekommt man »manchmal « nicht mehr und länger Geld, wenn man die fachlichen Ziele nicht erreicht und den Klienten behalten darf?

\section{Sechstes Kriterium: Effizienzgewinne}

Bei der Umstellung des Systems auf eine neue Finanzierungsform wird vieles besser. Aber auch wenn neue Finanzierungsformen die Effizienz der Organi- sation (oder des ganzen Systems) verbessern, müssen die durch Reformen entstehenden Transaktionskosten (Informationskosten, Änderungskosten, Vertragskosten etc.) höher sein als die damit verbundenen Transaktionskosten.

\section{Siebtes Kriterium: Bürokratie-, Qualitäts- und Kontrollkosten}

Umso individualisierter, umso bedarfsgerechter, umso nachhaltiger und qualitativer die Finanzierungsform ausgestaltet sein soll, desto energischer wachsen in der Regel die damit verbundenen Bürokratie-, Qualitäts- und Kontrollkosten. Schon heute investieren Fachkräfte in sozialen Einrichtungen ein Drittel ihrer Arbeitszeit in »indirekte Arbeitszeiten «. Neue, gute Finanzierungsformen haben häufig ihre Schattenseite im Dokumentationsaufwand, Prüfaufwand, Kontrollaufwand und einem Qualitätsmanagementsystem, das seinen Grenznutzen schon übertroffen hat. deutschen Sozialwesen praktizierten Finanzierungsformen wirken eher innovationsbremsend, eher produktivitätszementierend und stabilisieren vorhandene Dienstleistungsmodelle. Wenn »Pioniergewinne " vom Finanzierungsträger mit hoher Wahrscheinlichkeit in der nächsten Entgelt-Verhandlungsrunde kassiert werden, bleibt das System eher statisch.

\section{Zehntes Kriterium: Vermiedene Opportunitätskosten und Opportunitätsgewinne}

Jede soziale Einrichtung kostet Geld, aber jede soziale Einrichtung vermeidet auch immer die Kosten eines anderen Typs sozialer Einrichtung, in der ein Klient nicht ist. Wenn Finanzierungsformen nun so gestrickt sind, dass Anreizen, eine teurere Dienstleistungsform zu wählen, entgegen gewirkt wird, produzieren Finanzierungsformen nicht nur Kosten, sondern auch vermiedene

\section{"Es hat den Anschein, dass hinter den Rücken der Beteiligten die Regeln verändert wurden«}

\section{Achtes Kriterium: \\ Klientenbezogene \\ Dienstleistungsqualität}

Welcher Qualitätsbegriff wird durch die Finanzierungsform gefördert oder gebremst? Klammert sich die Finanzierungsform eher an operationalisierbare Kriterien der Strukturqualität, an administrativ überprüfbare Strukturmerkmale wie Quadratmeter, Personalquote und Fluchtwegkennzeichnungen oder an Merkmale der Lebensqualität bei den Klienten?

\section{Neuntes Kriterium: Dienstleistungsproduktivität und Pioniergewinne}

Dienstleistungsproduktivität verbessert sich durch die Verbesserung der Dienstleistungskonfiguration, durch bessere Kombinationen von Inputfaktoren und durch Innovationen, die bisherige »Produktionsfunktionen " deutlich übertreffen. Falls alle im
Kosten. Ebenso ermöglichen Finanzierungsformen mancher sozialen Dienstleistungen, die Angehörige und Klienten anstiften, erwerbstätig zu sein oder zu werden, mit der Folge von volkswirtschaftlichen Effekten durch Steuer- und Beitragszahlungen.

\section{Elftes Kriterium: Zitronenmarkt}

Wenn individualisierte Finanzierungsformen den Einkauf sozialer Dienstleistungen durch den Klienten ermöglichen, und wenn zwischen Klient und Anbieter eine deutliche Informationsassymetrie besteht, dann könnten diese an sich so begrüßenswerten individualisierten Finanzierungsformen einen $\mathrm{Zi}$ tronenmarkt fördern, auf dem sich zunehmend halbseriöse Anbieter tummeln, die davon profitieren, dass der Klient die Leistungsqualität der Anbieter letztlich nicht gut genug beurteilen kann. Die guten Anbieter haben dann die schlechten Karten und ziehen sich aus dem Markt zurück. 